\title{
Effet de l'alimentation séparée à base des feuilles de manioc post- récolte sur les performances des poulets de chair en finition
}

Joseph Rusty GUEMBO ${ }^{1}$, Prudence Pitchou ADZONA², Jean Bamard BATI ${ }^{3}$, Alain Juste SABOUKOULOU $^{1}$, Vincy Midrak NTSOUMOU ${ }^{1}$, Bienvenu Balthazar MABANZA MBANZA $^{3}$, Pachellie Edvylia PEPAH ${ }^{2}$, Fiacre Arnaud NDINGA ${ }^{3}$, Jean Luc HORNICK ${ }^{4}$ et Henri BANGA-MBOKO ${ }^{2-3 *}$

\author{
${ }^{1}$ Faculté des Sciences et Techniques, Université Marien NGOUABI, Brazzaville, Congo. \\ ${ }^{2}$ Ecole Nationale Supérieure d'Agronomie et de Foresterie, Université Marien NGOUABI, B.P 69 Brazzaville, \\ Congo. \\ ${ }^{3}$ Institut national de Recherche Agronomique (IRA), Avenue des premiers jeux africains face stade Alphonse \\ Massamba Débat, Brazzaville, Congo. \\ ${ }^{4}$ Institut Vétérinaire Tropical, Faculté de Médecine Vétérinaire, Université de Liège, Quartier Vallée, 2 Avenue \\ de Cureghem 7A-7D, 4000 Liège, Belgique. \\ *Auteur correspondant ; E-mail : hbangamboko@gmail.com, Téléphone : +242066851476 ou +242055778025
}

\section{REMERCIEMENTS}

Cette étude a reçu le soutien financier de la coopérative AGRO 4 Production, Congo.

\author{
Received: 14-05-2021
}

Accepted: $21-10-2021$

Published: 30-10-2021

\section{RESUME}

Les feuilles de manioc abandonnées sur les champs après la récolte des racines constituent une source locale en éléments nutritifs chez les animaux domestiques. Des technologies innovantes comme l'alimentation séparée à base de feuilles de manioc peuvent améliorer les performances des poulets de chair. L'alimentation séparée est une technique qui consiste à dissocier le régime alimentaire en deux fractions pour permettre à la volaille d'ajuster elle-même l'ingéré alimentaire en fonction de ses besoins journaliers. L'objectif de ce travail était de comparer l'alimentation mélangée et séparée à base de feuilles de manioc sur les performances des poulets de chair. Pour atteindre cet objectif, un échantillon de 100 poulets de chair de souche COBB 500 âgés de 3 semaines a été réparti en deux lots (témoin et traité). Chaque lot était subdivisé en 5 répétions de 10 poulets chacun. Les sujets du lot témoin ont été nourris avec un aliment contenant $5 \%$ de farine de feuilles de manioc servie dans une seule mangeoire tandis que ceux du lot traité ont reçu un aliment de base distribué dans une première mangeoire et de la farine de feuilles de manioc servie dans une seconde mangeoire. Les résultats ont montré que l'alimentation séparée des feuilles de manioc a amélioré significativement $((p<0.05)$ le poids vif final à 6 semaines (1692 g contre $1900 \mathrm{~g})$, la consommation de l'aliment de base (120,5 g contre 153,94), le $\operatorname{GMQ}(43,2$ g/jour contre 62,9 g/jour, l'indice de consommation (2,3 contre 2, 0). Par ailleurs, la consommation de la farine de manioc a été modulée en alimentation séparée et constante en alimentation mélangée $((p<0.05)$ ( $5 \mathrm{~g}$ par jour contre 1,3 g). De même, le traitement a réduit le coût de production du poulet de chair. L'incorporation des feuilles de manioc en mode séparé ouvre des perspectives intéressantes en aviculture fermière tropicale.

(C) 2021 International Formulae Group. All rights reserved.

Mots clés : Provende, Cobb 500, Cassava, croissance, coût de production, Congo. 


\title{
Effect of cassava leaf wastes meal by using a separate feeding on the performance of broiler chicken in the finishing stage
}

\begin{abstract}
Leave of cassava left on the fields after the harvest of the roots constitute a local source of nutritive elements for farm animals. By using a separated feeding, we hypothesized that performances of chicken may be improved. Therefore, the objective of the study was to evaluate the effect of a separated feeding on chicken in finishing stage. To check this assumption, a sample of one hundred COBB 500 chicken 3 week- old was randomized and divided into two groups (control and treated.) Each group was subdivided into 5 repeated groups of 10 chickens each one. The first group received a mixture of feed containing $5 \%$ of waste of leave given in a single throughout whereas the second group received a basic feeding without waste of leaves distributed in a first throughout and leaves of cassava served in the second throughout. Results showed that the separate feed significantly $(p<0$. 05) improved the final live weight at 6 weeks (1692 g versus $1900 \mathrm{~g})$, the consumption of basic food (120. $5 \mathrm{~g}$ versus 153. 94), the BGW (43.2 g/day versus $62.9 \mathrm{~g} / \mathrm{day})$, the index of consumption (2, 3 versus 2,0 ). In addition, the consumption of the flour of manioc was modulated in separate food and constant in mixed food (5 g per day against 1. 3). In the same way, the treatment reduced the production costs of chicken. The incorporation of the wastes of cassava as a local energy and protein sources by using separate feeding opens interesting prospects in tropical poultry farming.
\end{abstract}

(C) 2021 International Formulae Group. All rights reserved.

Keywords: Feed, Cobb 500, Manihot esculenta, growth, production costs, Congo.

\section{INTRODUCTION}

Le manioc originaire de l'Amérique du Sud est cultivé sous les tropiques pour ses racines tubérisées et les feuilles en alimentation humaine. Les pertes post-récolte génèrent une grande quantité de déchets notamment les épluchures, les eaux usées, les tiges et feuilles. Les feuilles sont des résidus riches en protéines et autres nutriments. Les feuilles de manioc contiennent des quantités importantes de protéines $(17,7 \%$ à $38,1 \%)$ en poids sec (Nassy et al., 2020).

Le rendement des feuilles après la récolte des racines peut alors atteindre 4, 6 tonnes de matières sèches par hectare (Regnier, 2011; Nassy et al., 2020). Cette biomasse foliaire constitue une source non négligeable pour l'alimentation animale. En Afrique tropicale, les feuilles sont utilisées comme alimentation pour les volailles (Eruvbetine et al., 2003 ; Ironkwe et al., 2012 ; Lukuyu et al., 2014 ; Noviadji et al., 2016). Mais, les données rapportées ne font pas l'unanimité des auteurs.

En effet, plusieurs travaux ont été menés avec succès sur la farine de feuilles de manioc comme ingrédient dans l'alimentation des cobayes et de la volaille (Iheukwumere et al.,2008 ; Dahouda et al., 2009 ; Noviadi et al., 2016 ; Mweugang et al., 2016). Ces auteurs soulignent l'importance de cette ressource alimentaire non conventionnelle comme une alternative efficace d'énergie et de protéines. Les feuilles peuvent être servies aux animaux sous forme fraîche ou séchée. Les feuilles séchées peuvent ainsi être incorporées dans l'alimentation des volailles jusqu'à un taux de $5 \%$ sans affecter leur croissance ni leurs performances (Houndonougbo et al., 2012a).

En revanche, les travaux antérieurs ont montré que l'incorporation jusqu'à 5\% de poudre de feuilles de manioc dans la ration a un effet négatif sur le gain moyen quotidien (GMQ), la consommation d'aliment et l'indice de consommation (Akinfala et al., 2002; Olajide et al., 2012 ; Adeyemi, 2013).

Le taux d'incorporation de la poudre de feuille de manioc dans les rations à plus de 5\% du régime alimentaire entraine une diminution de poids vif, GMQ, IC et un taux élevé de mortalité qui peuvent être dus à la présence des fibres et à l'acide cyanidrique (Ankinfala et al., 2002 ; Fasuyi et Aletor, 2005 ; Iheukwumere et al., 2008 ; Abu et al., 2015). 
Bindelle et al. (2004) et Houndonougbo et al. (2012b) soulignent que l'incorporation de $5 \%$ à $10 \%$ de la poudre de feuilles de manioc dans des régimes alimentaires des animaux constitue un meilleur compromis entre le coût de la ration et les performances des animaux. Ces derniers auteurs ont justifié cette baisse des performances par la présence des facteurs antinutritionnels. Tous les travaux de recherche pré cités ont été conduits en alimentation classique où les feuilles de manioc ont été mélangées aux autres ingrédients. Or il est connu depuis fort longtemps que les poulets à trois semaines d'âge sont capables de sélectionner naturellement les différents ingrédients d'un régime, ce qui permet d'ajuster précisément la consommation pour un maintien énergétique constant (Baradaran et al., 2021).

Le régime alimentaire séparé ou fractionné comprend plusieurs variables chez la volaille (chair et oufs) : la séparation des matières énergétiques des ingrédients riches en MAT et en minéraux a été préconisée (BangaMboko et al., 2007 ; Adzona et Banga-Mboko, 2017 ; Adzona et al., 2019) ainsi que la séparation d'un ingrédient d'autres composantes de la ration (Carré, 2000; Mantsanga et al., 2016) et l'alimentation séquentielle (Bouvarel et al., 2004).

Par contre, les données sur la séparation des feuilles de manioc avec d'autres ingrédients n'ont pas encore été expérimentées.

L'hypothèse de ce travail est que l'incorporation des feuilles de manioc dans une ration de volaille en mode séparé améliore les performances zootechniques du poulet de chair en finition.

L'objectif spécifique de ce travail est d'évaluer les performances des poulets de chair nourris à base des feuilles de manioc séparées des autres ingrédients.

\section{MATERIEL ET METHODES}

\section{La zone d'étude}

Les feuilles de manioc ont été collectées dans le Département du Pool, District de Ngabé. L'expérimentation a été réalisée dans les animaleries de l'IRA Brazzaville. Brazzaville capitale politique de la République du Congo, située au sud du pays sur la rive droite du fleuve Congo s'étend sur une superficie de $341821 \mathrm{Km}^{2}$ et compte une population de près de 1.174000 habitants soit $33 \%$ de l'ensemble de la population congolaise. Brazzaville s'étend sur une superficie de $110 \mathrm{Km}$, à $314 \mathrm{~m}$ d'altitude et est située entre $4^{\circ} 15^{\prime}$ latitude Sud et $15^{\circ} 14^{\prime}$ longitude Est. Elle est sous l'influence d'un climat du type Subtropical caractérisé par deux grandes saisons: une saison des pluies (d'octobre à mai) et une saison sèche marquée par une absence des pluies de juin à septembre. La température moyenne oscille entre $24,2^{\circ} \mathrm{C}$ et $26,4^{\circ} \mathrm{C}$ (janvier, février, mars et avril).

\section{Matériel végétal}

Les feuilles de manioc utilisées ont été prélevées dans les champs expérimentaux de la station de recherche Agronomique d'Odziba située à $100 \mathrm{~km}$ de Brazzaville sur la route nationale $\mathrm{n}^{\circ} 2$. Dans cette station, plusieurs variétés locales de manioc sont inventoriées et caractérisées (Otabo et al., 2016). Détachées des pétioles le jour de la récolte des tubercules de manioc de 12 mois d'âge, les feuilles ont été nettoyées puis lavées, moulues dans un broyeur à lame rotative couramment utilisé à la place du mortier pour le broyage des feuilles de manioc destinées à l'alimentation humaine. Le broyat a été cuit à un feu en bois pendant 60 minutes jusqu'à ce qu'à ce que les feuilles perdent leur couleur verte.

Après refroidissement, elles ont été séchées au soleil jusqu'à constater leur craquement au toucher. Le produit sec a été emballé dans les sacs en polypropylène. Des échantillons ont été prélevés pour l'analyse chimique. Les résultats de l'analyse sont consignés dans le Tableau 1.

\section{Le matériel animal et les conditions pré expérimentales}

Cent (100 poussins) d'un jour de souche COBB 500 en provenance de Belgique ont été élevés au sol sur litière de menuiserie jusqu'à l'âge de 3 semaines. Pendant une phase de démarrage d'une semaine, les poussins ont été élevés dans une poussinière, à une densité de 
20 poussins au $\mathrm{m}^{2}$ et nourris avec un aliment contenant $2900 \mathrm{kcal}$ par $\mathrm{Kg}$ et $22 \%$ MAT. A l'âge de 7 jours, les poussins ont été transférés dans un poulailler de croissance dont la densité a été 15 sujets au $\mathrm{m}^{2}$. Ils ont été nourris avec un aliment contenant $3000 \mathrm{kcal} / \mathrm{kg}$ et $20 \%$ MAT. L'eau a été distribuée à volonté, la température et l'hygrométrie ont été contrôlées par un thermomètre mural et un hygromètre jusqu'à trois semaines.

\section{Dispositif expérimental}

Au jour 21, les poulets ont été répartis à poids égal en deux lots (témoin et traité) de 50 poussins chacun. Chaque lot a été subdivisé en 5 répétitions de 10 poulets chacun. Un seul régime a été expérimenté. Le dispositif est indiqué à la Figure 1.

Le lot témoin a reçu un aliment dans lequel la farine de feuilles manioc a été mélangée aux autres ingrédients (farine de feuilles de manioc mélangée ou FFMM), distribué dans une seule mangeoire (Figure1 B). Le lot traité était nourri avec un aliment de base servi dans une première mangeoire (sans la farine de feuilles de manioc) .et des feuilles de manioc distribuées dans une seconde mangeoire (farine de feuilles de manioc séparée ou FFMS) (Figure 1 A).

\section{Aliment et conduite alimentaire}

Les rations alimentaires sont indiquées au Tableau 2. Le Tableau 3 illustre la composition centésimale des rations expérimentales et leur valeur nutritionnelle. Les rations journalières étaient distribuées à volonté, les quantités d'aliment ont été pesées avant la distribution. Les pesées des refus ont permis de calculer les quantités d'aliment consommées par jour.

\section{Variables zootechniques mesurées \\ Taux de Mortalité}

Le taux de mortalité a été calculé par la formule suivante :

M

$=\frac{\text { Nombre de morts au cours de la periode }}{\text { Cheptel initial }}$
Poids vif

Les poulets de chaque lot ont tété pesés au jour 21 puis chaque semaine jusqu'au jour 42, à l'aide d'une balance électronique. Les données obtenues ont servi pour dresser la courbe de croissance pondérale.

\section{Consommation volontaire d'aliment de base}

La consommation volontaire alimentaire des poulets a été obtenue grâce aux pesées quotidiennes de la quantité d'aliment servie et non consommée, et calculée selon la formule :

CVA

Quantité d'aliment distribuée

$=\frac{\text { par jour }- \text { Quantité } \mathrm{d}^{\prime} \text { aliment refusée par jour }}{\text { Nombre de sujets }}$

La consommation volontaire de la farine de feuilles de manioc (CVFFM)

La consommation volontaire de la farine de feuilles de manioc du lot traité a été calculée par la seule différence entre les quantités distribuées et les quantités non consommées car la farine de feuilles de manioc était servie dans une même mangeoire.

Dans lot témoin la consommation volontaire de la farine de feuilles de manioc a été déduite de son taux d'incorporation dans le mélange, selon la formule suivante :

\section{CVFFM= $=($ consommation volntaire $=$ d'aliment consommé $x \mathbf{5}$ \\ 100}

\section{Consommation volontaire d'eau}

La consommation volontaire d'eau (CVE) a été calculée sur la base de la formule suivante:

$$
\mathrm{CVE}=\frac{\begin{array}{c}
\text { Quantitéd'eau distribuée par jour-Quantité } \\
\text { d'eau refusée par jour }
\end{array}}{\text { Nombre de sujets }}
$$

\section{Gain Moyen Quotidien (GMQ)}

Le gain moyen quotidien dans les deux lots était calculé sur la base de la formule suivante:

$$
\mathrm{GMQ}=\frac{\text { Poids vif final }- \text { poids vif initial }}{\text { Durée de la periode }}
$$

\section{Indice de consommation}

L'indice de consommation (I.C.) a été calculé selon la formule suivante :

Quantité moyenne d'aliment

$$
\text { IC }=\frac{\text { consommé en une semaine }}{\text { poids gagné en une semaine }}
$$




\section{Indicateurs économiques calculés}

L'évaluation économique a été faite sur la base du coût de production alimentaire. Ce coût a été calculé sur la base du prix des matières premières pratiqué sur le marché local pendant la période expérimentale. Pour la farine de feuilles de manioc, le prix a été estimé en tenant compte de différentes opérations effectuées de la récolte au séchage.

Les indicateurs ci-après ont été calculés :

\section{Le coût de production du kilogramme de poids vif (CPKV)}

Il a été obtenu en multipliant l'indice de consommation par le coût du kilogramme d'aliment.
Le coût alimentaire $(\mathrm{CAL}):$ Il a été calculé comme suit : $C A L=\frac{\text { aliment consommé }}{\text { Poids total }}$

La conversion alimentaire (CVAL) : C'est le poids vif moyen final - poids vif moyen final

\section{Analyse statistique}

Les données brutes ont été saisies sur Excel puis transférées dans le logiciel Statview 5.0 2000. La comparaison des moyennes entre le lot témoin et le lot traité a été effectuée avec le test $t$ de Student. Deux moyennes étaient statistiquement différentes quand la valeur de $\mathrm{P}$ était inférieure à 0,05 .

Tableau 1 : Analyse chimique de la farine de feuilles de Manihot esculenta.

\begin{tabular}{|c|c|c|c|}
\hline Test & Unité & Résultats & Méthodes \\
\hline Humidité $\left(103^{\circ} \mathrm{C}\right)$ & $\%$ & 8,8 & Règlement CE 152/2009 \\
\hline $\begin{array}{l}\text { Protéines brutes (Dumas, } \\
\text { expréqkj) }\end{array}$ & $\%$ & 25,8 & NF EN ISO 16634-1 \\
\hline Matière grasse A (éther pétrole) & $\%$ & 4,7 & Règlement CE 152/2009 \\
\hline Cellulose brute (Wende) & $\%$ & 11,7 & Règlement CE 152/2009 \\
\hline Cendres brutes & $\%$ & 5,8 & Règlement CE 152/2010 \\
\hline Calcium $(\mathrm{Ca})$ & $\%$ & 0,8 & ICP-OES Méthode interne \\
\hline \multirow[t]{2}{*}{ Phosphore (P) } & $\%$ & 0,25 & ICP-OES Méthode interne \\
\hline & $\begin{array}{l}\text { AMIN } \\
(\%)\end{array}$ & RAMME & \\
\hline Cystine & & 0,26 & \\
\hline Acide aspartique & & 1,74 & \\
\hline Proline & & 1,13 & \\
\hline Méthionine & & 0,36 & \\
\hline Thréonine & & 0,85 & \\
\hline Sérine & & 0,87 & \\
\hline Acide glutamique & & 2,47 & \\
\hline Glycine & & 0,95 & \\
\hline Alanine & & 1,06 & \\
\hline Valine & & 1,09 & \\
\hline Isoleucine & & 0,88 & \\
\hline Leucine & & 1,58 & \\
\hline Tyrosine & & 0,68 & \\
\hline Phénylalanine & & 1,01 & \\
\hline Lysine & & 1,12 & \\
\hline Histidine & & 0,42 & \\
\hline Arginine & & 1,03 & \\
\hline
\end{tabular}




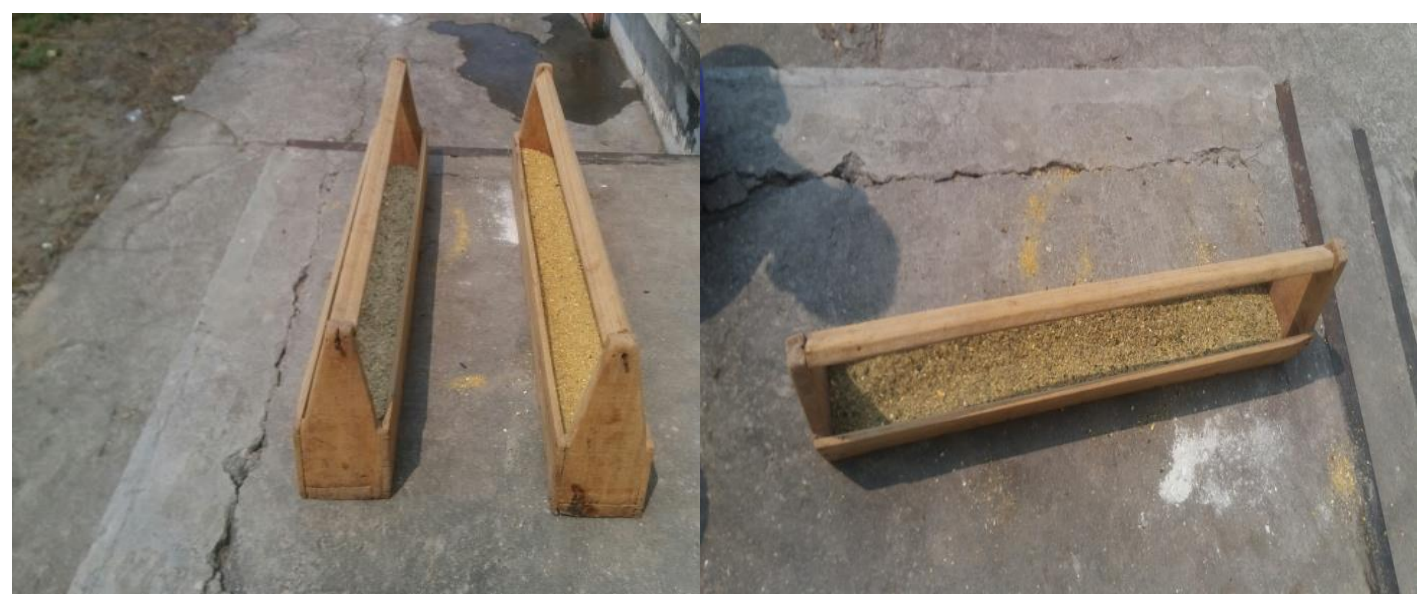

A

B

Figure 1 : Alimentation fractionnée.

Figure 1 A : La mangeoire de gauche contient uniquement de la farine des feuilles de manioc (FFM) tandis que celle de droite contient l'aliment de base (groupe traité).

Figure 1 B : Distribution de la ration alimentaire du lot témoin dans une seule mangeoire dans laquelle les feuilles de manioc ont été mélangées (FFMM) aux autres ingrédients.

Tableau 2 : Matières premières et composition nutritionnelle des aliments expérimentaux des poulets de chair en finition.

\begin{tabular}{|c|c|c|c|}
\hline \multicolumn{4}{|c|}{$\begin{array}{c}\text { Aliment finition (à partir de la } \\
\text { 4ème semaine) }\end{array}$} \\
\hline & FFMM & \multicolumn{2}{|r|}{ FFMS } \\
\hline Matières premières $(\%)$ & $\begin{array}{c}\text { En une } \\
\text { mangeoire }\end{array}$ & $\begin{array}{l}\text { Première } \\
\text { mangeoire }\end{array}$ & $\begin{array}{l}\text { Deuxième } \\
\text { mangeoire }\end{array}$ \\
\hline Maïs & 62,25 & 62,25 & 0 \\
\hline Son fin de blé & 3 & 3 & 0 \\
\hline Feuille de manioc & 5 & 0 & 5 \\
\hline Tourteau de soja & 20,05 & 20,05 & 0 \\
\hline Farine de poisson & 4 & 4 & 0 \\
\hline Méthionine & 0,1 & 0,1 & 0 \\
\hline Lysine & 0,1 & 0,1 & 0 \\
\hline Calcaire & 1,5 & 1,5 & 0 \\
\hline Prémix & 1 & 1 & 0 \\
\hline Huile de palme & 3 & 3 & 0 \\
\hline Sous total & 100 & 95 & 5 \\
\hline Total & 100 & & 100 \\
\hline \multicolumn{4}{|c|}{ Composition nutritionnelle } \\
\hline Protéine brute $(\%)$ & 18,05 & 16,95 & 1,1 \\
\hline E.M (Kcal/Kg MS) & 3169,2 & 2959,225 & 210 \\
\hline Méthionine (\%) & 0,45 & 0,38 & 0,065 \\
\hline
\end{tabular}




\begin{tabular}{lccc} 
Lysine $(\%)$ & 1,17 & 0,92 & 0,255 \\
Calcaire $(\%)$ & 0,9 & 0,86 & 0,0395 \\
Phosphore total $(\%)$ & 0,48 & 0,46 & 0,019 \\
\hline
\end{tabular}

Tableau 3. Effet du mode d'apport de la farine de feuilles de manioc sur quelques paramètres zootechniques des poulets de chair en finition.

\begin{tabular}{|c|c|c|c|c|}
\hline \multirow[b]{2}{*}{ Semaine } & \multicolumn{2}{|c|}{ FFMM } & \multicolumn{2}{|c|}{ FMMS } \\
\hline & $\mathrm{CAB}$ & CFM & $\mathrm{CAB}$ & CFM \\
\hline \multicolumn{5}{|c|}{ Consommation alimentaire individuelle journalière (g) } \\
\hline S4 & $94,7^{\mathrm{a}}$ & $4,95^{\mathrm{a}}$ & $102,1^{\mathrm{b}}$ & $2,1^{\mathrm{b}}$ \\
\hline S5 & $118,3^{\mathrm{a}}$ & 6,2 & $139,3^{\mathrm{a}}$ & $0,8^{\mathrm{a}}$ \\
\hline S6 & $120,4^{\mathrm{a}}$ & $6,34^{\mathrm{a}}$ & $153,9^{\mathrm{b}}$ & $1,1^{\mathrm{b}}$ \\
\hline \multicolumn{5}{|c|}{ Consommation volontaire d'eau (ml/j/sujet) } \\
\hline S4 & \multicolumn{2}{|c|}{$193,1 \pm 41,9^{\mathrm{a}}$} & \multicolumn{2}{|c|}{$213,2 \pm 66,5^{b}$} \\
\hline S5 & \multicolumn{2}{|c|}{$336,4 \pm 20,5^{\mathrm{a}}$} & \multicolumn{2}{|c|}{$361,904 \pm 36,6^{\mathrm{b}}$} \\
\hline S6 & \multicolumn{2}{|c|}{$339 \pm 36,6^{\mathrm{a}}$} & \multicolumn{2}{|c|}{$377,6 \pm 16,28^{b}$} \\
\hline & \multicolumn{4}{|c|}{ Gain moyen quotidien (g/j) } \\
\hline S4 & \multicolumn{2}{|l|}{$57,2^{\mathrm{a}}$} & \multicolumn{2}{|l|}{$67,2^{\mathrm{b}}$} \\
\hline S5 & \multicolumn{2}{|l|}{$55,6^{\mathrm{a}}$} & \multicolumn{2}{|l|}{$69,9^{\mathrm{b}}$} \\
\hline \multirow[t]{2}{*}{ S6 } & $43,2^{\mathrm{a}}$ & & $62,9^{\mathrm{b}}$ & \\
\hline & \multicolumn{4}{|c|}{ Indice de consommation } \\
\hline S4 & $1,7^{\mathrm{a}}$ & & $1,6^{\mathrm{b}}$ & \\
\hline S5 & $2,2^{\mathrm{a}}$ & & $2,0^{\mathrm{b}}$ & \\
\hline S6 & $2,9^{\mathrm{a}}$ & & $2,5^{\mathrm{b}}$ & \\
\hline
\end{tabular}

Légendes : FFMM : farine de feuilles de manioc mélangées ; FFMS : farine de feuilles de manioc séparées ; CAB :

Consommation de l'aliment de base ; CFM : consommation de la farine de feuilles de manioc.

\section{RESULTATS}

Effet du mode de présentation des feuilles de manioc sur la mortalité :

Un taux de mortalité de $3,66 \%$ a été observé dans les deux lots.

Effet du mode de présentation des feuilles de manioc sur la croissance pondérale

La Figure 2 illustre l'évolution du poids vif en fonction de l'âge. Dans les deux groupes le poids a évolué avec l'âge des oiseaux durant toute la période expérimentale. Toutefois, le traitement a eu un effet significatif sur le poids final de la cinquième à la sixième semaine
$(1900$ g contre 1692 g) à la fin de l'expérimentation $(\mathrm{p}<0,05)$.

Effet du mode de présentation des feuilles de manioc sur la consommation d'aliment, la consommation volontaire d'eau, le GMQ et l'IC.

Les résultats sont présentés dans le Tableau 4. L'effet sur la consommation volontaire d'aliment de base (sans le manioc) est en augmentation avec l'âge dans les deux groupes. Il y avait une différence significative entre les deux lots. Au cours des semaines 4, 5 et 6 , une augmentation respective de $(+7,6 \%)$, 
$(+18,3 \%),(+26,82 \%)$ a été relevée. La différence sur la consommation de l'aliment de base a été significative d'une semaine à une autre. La prise de l'aliment de base (sans feuilles de manioc) a été plus élevée en mode séparé soit $131,8 \mathrm{~g}$ contre $111,16 \mathrm{~g}$ dans le lot témoin.

En revanche, le traitement a induit une forte réduction de la consommation volontaire, individuelle et journalière des feuilles de manioc. Les pourcentages de réduction enregistrés étaient de (-67\%), $(-81 \%),(-82 \%)$ respectivement de la première, deuxième et troisième semaine.Cette consommation a été constante au cours des deux dernières semaines. Il ressort que la consommation des feuilles de manioc a été plus élevée chez les poulets nourris avec FFMM que FFMS. La consommation volontaire d'eau en mode séparé a augmenté significativement de $+19 \%$ à la $4{ }^{\text {ème }}$ semaine, et de $+11,7 \%$ à la $6^{\text {èm }}$ semaine dans le groupe ayant reçu le traitement $(\mathrm{P}<0,05)$.

Les données montrent que l'alimentation en mode séparé, a amélioré significativement la vitesse de croissance. Au cours de trois semaines de l'essai, elle a augmenté de $+17,5 \%,+25,71 \%$ et $+45,6 \%$ respectivement à la $4^{\text {ème }}, 5^{\text {ème }}$ et $6^{\text {ème }}$ semaine. Enfin, l'ingestion élevée des feuilles de manioc en mode séparé a amélioré l'indice de conversion alimentaire.

Effet du mode de présentation des feuilles de manioc sur les indicateurs économiques

Les données sont consignées dans le Tableau 4. Le traitement a amélioré le coût de production du $\mathrm{kg}$ de poids vif final, le coût de production du $\mathrm{kg}$ d'aliment et la conversion alimentaire.

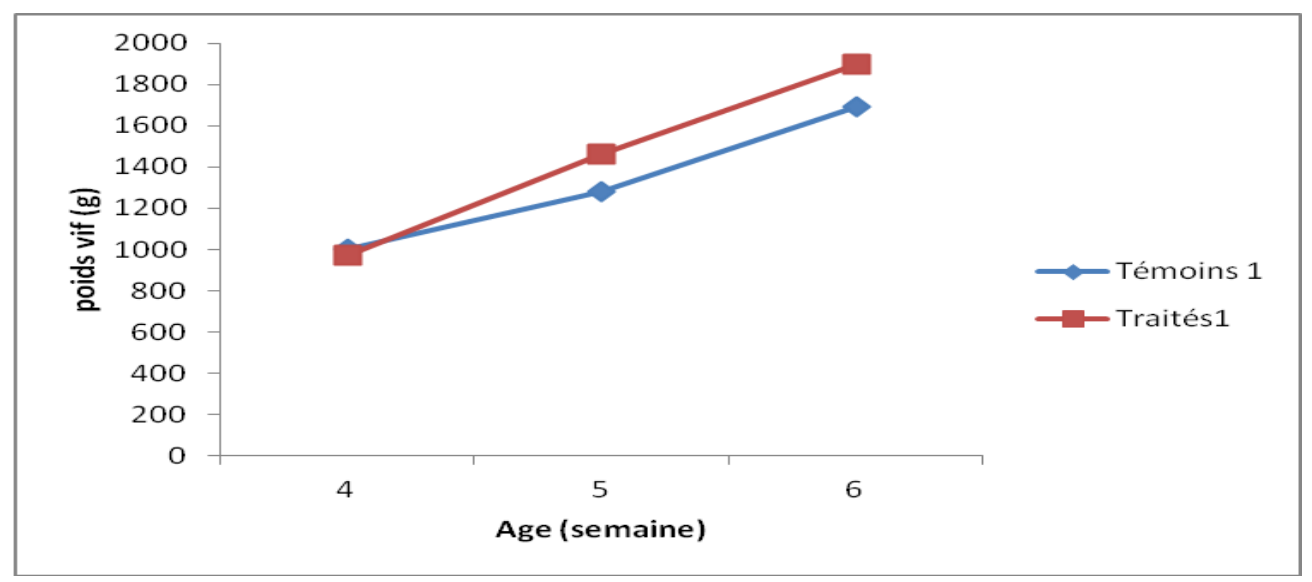

Figure 2 : Effet du mode d'apport de la farine de feuilles de manioc sur la croissance pondérale des poulets de chair en finition.

Tableau 4 : Effet du mode d'apport de la farine de feuilles de manioc sur les paramètres économiques des poulets de chair en finition.

\begin{tabular}{lcc}
\hline & FMMM & FMMS \\
\hline $\begin{array}{l}\text { Prix aliment } \\
\text { (FCFA/Kg) }\end{array}$ & 302,0284 & 302,0284 \\
Coût de production du Kg PV (ICxPrix aliment) & 694,66 & 605,06 \\
& 2,261 & 2,208 \\
Coût alimentaire (aliment consommé/poids total) & 1092,5 & 1400,6 \\
\hline
\end{tabular}




\section{DISCUSSION}

\section{Effets du mode de présentation des feuilles de manioc sur la mortalité}

Le traitement n'a pas eu un effet négatif sur la mortalité dont le taux n'a pas dépassé $10 \%$ en fin d'expérience, ce qui prouve que le traitement n'a pas eu d'effet nocif et justifie une bonne conduite des oiseaux. Ce taux est plus élevé par rapport aux résultats rapportés par Olajide et al. (2012) où aucune mortalité n'a été signalée. Ce faible taux de mortalité dans la présente étude pourrait se justifier par l'effet de la cuisson préalable des feuilles de manioc car le traitement à la chaleur a certainement réduit le taux de l'acide cyanhydrique dans les feuilles, le taux des facteurs antinutritionnels tels que les tanins et autres, le taux de fibres alimentaires qui respectent la norme (Regnier et al., 2011 ; Houndonougbo et al., 2012b ). La teneur en cyanure (300 et $1500 \mathrm{mg}$ de $\mathrm{HCN} / \mathrm{kg}$ de poids frais au niveau des feuilles) et en tanins, les feuilles fraîches, sèche ou cuite ne peuvent être données aux poulets qu'en quantités très limitées. Le hachis des feuilles peut également être séché au soleil soit cuit ou ensilé pour éliminer l'essentiel du cyanure, ce qui augmente l'appétence de l'animal et rendent les feuilles consommables sans risque (Adeyemi, 2013).

\section{Effets du mode de présentation des feuilles de manioc sur la consommation volontaire de la farine de feuilles de manioc}

Dans cette étude, c'est uniquement la farine de feuilles de manioc mélangées (FFMM) qui a été séparée de tous les autres ingrédients afin d'apprécier le comportement de consommation volontaire des poulets de chair. L'expérimentation a prouvé évidemment que les feuilles de manioc présentées à part des autres ingrédients ont été faiblement consommées. De tels résultats ont été rapportés chez les poules pondeuses soumises à une alimentation calcique séparée (Banga Mboko et al., 2007 ; Mantsanga et al., 2016). Ceci révèle que les feuilles de manioc ne sont pas appétibles chez le poulet de chair soumis à une alimentation conventionnelle car les individus soumis à une consommation volontaire des feuilles servies dans une autre mangeoire ont montré une meilleure utilisation en fonction de l'âge.

Cette façon de moduler la consommation des feuilles de manioc en mode séparé est permet d'améliorer les performances zootechniques et économiques observées dans la présente étude.

\section{Effets du mode de présentation des feuilles} de manioc sur la consommation volontaire de l'aliment de base

L'aliment de base (sans feuilles de manioc) a été majoritairement consommé par les poulets en finition dans le groupe traité. Ce comportement peut être expliqué par le fait que l'appétit des oiseaux est d'abord étroitement lié à leurs besoins énergétiques. Parmi les caractéristiques de l'aliment qui modifient le plus la consommation sont entre autres la concentration énergétique. En effet, l'animal cherche en priorité à ingérer la quantité d'aliment lui permettant de couvrir ses besoins énergétiques (Houndonougbo et al., 2012a).

En mélange, même si les oiseaux sont réputés être grands trieurs, ils n'ont pas pu échapper au prélèvement des feuilles de manioc. L'inappétence observée pourrait donc justifier la faible consommation constatée chez les sujets soumis à la farine de feuilles de manioc séparées (FFMS) que chez ceux soumis à la farine de feuilles de manioc mélangées (FFMM). Ce qui d'ailleurs témoigne la réduction de l'appétit des sujets du lot traité.

Antérieurement, la réduction de la prise d'aliment chez les poulets a été constatée quand la FFM a été mélangée à $5 \%$ à d'autres ingrédients (Olajide, 2012; Adeyemi et al., 2013).

On pourrait accuser certains éléments qui réduisent la consommation d'un aliment. En effet, les oiseaux sont sensibles aux formes ; ils doivent s'habituer par exemple d'un aliment granulés à un aliment farineux soulignent Carré (2000). Ces auteurs rapportent que la texture ainsi que la granulométrie de l'aliment peuvent 
influencer la consommation ; la faible taille des particules la diminue. dans cette étude tous les ingrédients ont été présentés en fines particules, bien que le cyanure présent dans les feuilles de manioc est éliminé par le traitement à la chaleur (Regnier et al., 2011 ; Lukuyu et al., 2014). Par ailleurs, les facteurs antinutritionnels tout comme les carences en certains nutriments tels que les acides aminés, peuvent modifier l'ingestion de l'aliment. Par contre, chez Iheukwumere et al. (2008) qui ont substitué des feuilles de manioc au pourcentage de $10 \%$, la consommation est plus élevée (148 g/sujet).

Ces variations peuvent être expliquées non seulement par les conditions d'élevage, mais aussi par les différences géographiques, la saison, la diversité ou la variabilité génétique existant dans cette population des volailles et des feuilles de manioc (Dahouda et al., 2009).

Un aliment riche en fibres comme la FFM est susceptible de réduire la consommation. En effet, des composés inertes tels que les fibres végétales impactent sur des signaux de satiété situés au niveau du jabot et du reste du tube digestif (Bouvarel et al., 2004). Cependant, une consommation élevée de la fraction énergétique a été notée par Adzona et al., (2019) qui ont incorporé des aliments riches en fibres à $13 \%$ dans la ration des poulets de chair COBB 500 en finition.

Effets du mode de présentation des feuilles de manioc sur la consommation volontaire d'eau.

Le traitement a eu un effet significatif sur la consommation d'eau. Ce constat concorde avec les résultats de plusieurs études sur le régime alimentaire en mode séparé qui induit une augmentation de la consommation d'eau chez la volaille (Bouvarel et al., 2004 ; Banga-Mboko et al., 2007 ; Adzona et BangaMboko., 2017).

\section{Effets du mode de présentation des feuilles de manioc sur la croissance pondérale}

La croissance pondérale dans cette étude est similaire à celle rapportée par Fasuyi et Aletor (2005) lorsqu'ils ont étudié l'effet du remplacement de maïs avec un mélange de racine et de feuilles de manioc à un taux de $50 \%$ sur les performances des poulets. Toutefois, ces chercheurs n'ont observé aucune différence significative, contrairement aux effets mesurés dans la présente étude. Cependant, les résultats sur le poids vifs sont meilleurs à ceux obtenus par Dahouda et al., (2009) lorsqu'ils ont évalué en finition. Par contre, les résultats de la présente étude sont inférieurs à ceux des travaux réalisés par Abu et al., (2015).

Toutefois la performance est généralement maintenue avec des faibles niveaux de farine de feuilles de manioc (Trompiz et al., 2007 ; Iheukwumere et al., 2007). Cependant, un effet contraire est observé quelquefois avec des niveaux audessus de 5\% (Ankinfala et al., 2002). Certains auteurs ont constaté une performance acceptable avec des valeurs élevées de feuilles de manioc (10 à 20\%). Toute la farine végétale du manioc composée des feuilles et racine a aussi été testée et les résultats sont similaires dans une introduction proportionnelle à la farine de feuilles de manioc dans une alimentation complète, c'est-à-dire dégradation de la performance de production (Akinfala et al., 2002 ; Eruvbetine et al., 2003).

Effets du mode de présentation des feuilles de manioc sur le Gain moyen quotidien (GMQ)

Les résultats de cette étude se corroborent ceux de Ironkwe et al., (2012) qui affirment que l'incorporation des feuilles de manioc à $5 \%$ dans la ration n'affecte pas le gain de poids.

En outre, le GMQ dans cette étude etait plus élevé par rapport à ceux observés par Olajide et al., (2012) (25 g/j - $40 \mathrm{~g} / \mathrm{j})$; Abu et al., (2015) $(37,3 \mathrm{~g} / \mathrm{j}-41,1 \mathrm{~g} / \mathrm{j})$.

Effets du mode de présentation des feuilles de manioc sur l'Indice de consommation (IC)

La réduction significative de l'IC observée est une conséquence de la bonne consommation d'aliment comme le témoigne 
les valeurs élevées du GMQ. Le traitement a amélioré l'IC des poulets. Ces résultats se rapproche de ceux rapportés par Ironkwe et al., (2012). Cependant, cette observation est contradictoire à celle de Dahouda et al., (2009) qui ont révélé que la consommation d'aliment ayant été proportionnelle au gain de poids, aucune différence significative n'a été observée sur l'IC. Chez Iheukwumere et al. (2008) l'IC n'est pas intéressante car les valeurs sont de l'ordre de 3,7- 4,78. A contrario, Noviadi et al. (2016) ont obtenu des meilleurs résultats par apport à la présente étude $(1,43-1,3)$ lorsqu'ils ont incorporé les feuilles de manioc à $7 \%$ en mode mélangé.

\section{Effet du mode de présentation sur l'analyse économique}

Dans cette étude, les feuilles de manioc en mode séparé ont induit une diminution du coût de production du $\mathrm{kg}$ du poids vif du poulet .Ces résultats sont similaires avec les travaux antérieurs qui ont rapporté que l'incorporation de la FFM a eu une incidence économique positive (Dahouda et al., 2009). Houndonougbo et al., (2012b) ont obtenu une réduction de 12 à $20 \mathrm{~F} \mathrm{CFA} / \mathrm{Kg}$ du prix du $\mathrm{Kg}$ d'aliment chez les pondeuses Isa Brown.

\section{Conclusion}

Cette étude avait pour objectif d'évaluer l'effet d'une ration contenant des feuilles de manioc distribuées séparément de l'aliment de base. Il est apparu que toutes les variables contrôlées ont été en faveur de l'alimentation séparée. Cette étude apporte de nouvelles connaissances sur l'utilisation des feuilles de manioc chez le poulet de chair. Ainsi, la farine de feuilles constitue une source alternative d'énergie et de protéines brutes en particulier au cours de la période de finition.

\section{CONFLIT D'INTERETS.}

Les auteurs déclarent l'existence d'aucun conflit d'intérêts.

\section{CONTRIBUTIONS DES AUTEURS}

JRN et PEP ont a réalisé l'expérimentation, le suivi était assuré par PPA et JBB, VMN et BBM ont mis au point les formulations des rations alimentaires, FAN a fabriqué les aliments destinés à l'expérimentation et le suivi des oiseaux les week-ends et jours fériés, AJS a participé à la collecte des données et le traitement statistique, JLH a supervisé les analyses chimiques des échantillons en Belgique et enfin HBB a supervisé toute l'étude.

\section{REMERCIEMENTS}

Les auteurs expriment leur gratitude à l'Institut national de Recherche Agronomique (IRA) du Congo pour avoir accepté d'abriter l'expérimentation.

\section{REFERENCES}

Abu OA, Olaleru I F, Oke TD, Adepegba VA, Usman B. 2015. Performance of broiler chicken fed diets containing cassava peel and leaf meals as replacements for maize and soya bean meal. Int. J. Sci. Tech, 4(4): 169-172. DOI: $10.9790 / 2380-08324146$

Adeyemi O A, Jimoh B, Olufade OO. 2013. Soybean meal replacement with cassava leaf: blood meal mix with or without enzyme in broiler diets: Arch. Zootech., 62(238): 275-285.

Adzona PP, Bonouga, Bati JB, Ndinga FA, Onzomoko LD, Itoua PL, Kiki PS, Dotchet IO, Banga-Mboko H, Youssao A K. 2019. Influence du tourteau de sésame en alimentation fractionnée séparée et séquentielle sur les performances zootechnique et économique du poulet de chair standard de la souche Coob 500. Rev.Int. Sci. App., 2 (1) : 1-11.

Adzona PP, Banga-Mboko H. 2017. Effect of separating energetic feedstuffs in the finisher diet on performance of common guinea fowl (Numida meleagris 1.1758) under tropical climate. J. Anim. Health. Prod., 5 (4): 143-148.

Ankinfala EO, Aderibigbe AO, Mantannri O. 2002. Evaluation of the nutritive value of whole cassava plant as replacement for maize in starter diets for broiler chicken. Liv.Res. Rural Dev., 14 (-6):23-30. 
Banga-Mboko, Mabanza-Mbanza BB, Adzona PP, Batessana C. 2007. Réponse à l'alimentation calcique séparée de lignées commerciales de poules pondeuses Shaver sous les conditions tropicales du Congo Brazzaville. Bul. $f$ Anim. Prod. Heath in Africa, 55: 43-50.

Baradaran N, Shahir MH, Taheri HR, Bedford MR. 2021. Effect of sequential feeding of phosphorus-deficient diets and high-dose phytase on efficient phosphorus utilization in broiler chickens. Live Sci. https://doi.org/10.1016/j.livsci.2020.104 368 (in press).

Bindelle J, Buldgen A. 2004. Utilisation des plantes à tubercules ou à racines tubéreuses en alimentation animale. Troupeaux et Cultures des Tropiques, (4) : 47-50.

Bouvarel I, Barrier-Guillot B, Larroude P, Boutten B, Leterrier C, Merlet F, Vilarino M, Roffidal L, Tesseraud S, Castaing J, Picard M. 2004. Sequential feeding programs for broiler chickens: Twenty-four- and forty-eight-hour cycles. Poult. Sci., 83: 49-60.

Carré B. 2000. Effets de la taille des particules alimentaires sur les processus digestifs chez les oiseaux d'élevage. Productions Animales, INRA, 12(2): 31-136. 〈hal02696013>

Dahouda M, Toleba S, Youssao A, Mama AAA, Ahounou S, Hornick JL. 2009. Utilisation des cossettes et des feuilles de manioc en finition des pintades (Numidameleagris): performances zootechniques, coûts de production, caractéristiques de la carcasse et qualité de la viande. Ann. Méd.Vét., l.1 (53): 8287.

Eruvbetine D, Tajudeen ID, Adeosun AT, Olojedde AA. 2003. Cassava (Manihot esculenta) leaf and tuber concentrate in diet for broiler chickens. Biores. Technol., 86 : 277-281.

Fasuyi AO, Aletor VA. 2005. Protein Replacement Value of Cassava (Manihot esculenta, Crantz) Leaf Protein
Concentrate (CLPC) in Broiler Starter: Effect on Performance, Muscle Growth, Haematology and Serum Metabolites. International Journal of Poultry Science, 4(5): 339-349.

Houndonougbo MF, Chrysostome CA, Houndonougbo VP. 2012a. Performances de ponte et qualité des œufs des poules pondeuses ISA Brown alimentées avec des rations à base de feuilles séchées de manioc (Manihot esculenta, Crantz). Int. J. Biol. Chem. Sci., 6(5): 1950-1959.

Houndonougbo MF, Chrysostome C, Houndonougbo VP. 2012b. Performances bioéconomiques des poulettes alimentées avec des rations à base de feuilles séchées de manioc (Manihot esculenta). Int. J. Biol. Chem. Sci, 6(2) : 670-676.

Iheukwumere FC, Ndubuisi EC, Mazi EA, Onyekwere MU. 2007. Crowth,blood chemistry and carcass yield of Broilers fed cassava leaf meal (Manihot esculenta Crantz). Int. J. Poult. Sci.6(8): 555-559.

Ilheulkwumere FC, Ndubuisi EC, Mazi EA, Onyenkwere MU.2008. Performance, nutrient utilization and organ characteristics of broilers fed cassava leaf meal (Manihot esculenta Crantz). Parkistan J. Nutr., 7 (1):13-16.

Ironkwe MO, Ukanwoko AI, 2012. Growth performance of broiler finisher birds fed composite cassava meal (CCM). Bull. Environ. Pharmacol. Life Sci.,1(6): 30 34.

Lukuyu B, Iheanacho O, Duncan A, Beveridge M, Blümmel M. 2014. Use of cassava in livestock and aquaculture feeding programs. Int. Livest. Res. Inst.,25: 23-26.

Mantsanga HB, Amona I, Banga-Mboko H, Bakana1 MA, Adzona PP. 2016. Effet de l'alimentation calcique séparée sur les performances de production de la poule Lhoman Brown sous climat tropical humide J. Appl. Biosci.,97: 9212 -921.

Nassy MLM, Hauser S, Egwekhide M, Batawila K, Kulakow P, Abberton M. 2020. Rendement en feuilles et racines de trois variétés améliorées de manioc 
(Manihot esculenta Crantz) en réponse aux fertilisants organo-minéraux et à la récolte des feuilles au Sud-Ouest du Nigeria. Int. J. Biol. Chem. Sci., 14(4): 1432-1447.

Mweugang NN, Tendonkeng F, Miegoue E, Matumuini FEN, Zougou GT, Fonteh FA, Boukila B, Pamo ET. 2016. Effets de l'inclusion de feuilles de manioc (Manihot esculenta Crantz) dans la ration sur les performances de reproduction du cobaye (Cavia porcellus L.) local camerounais. Int. J. Biol. Chem. Sci., 10(1): 269-280.

Noviadi R, Candra AA. 2016. Effect of pellet containing cassava leaves meal at various particle sizes on broiler R. Bangladesh Soc. Vet. Med., 14 (1): 9-13.

Olajide AA, Richard AS. 2012. Performance of broiler chickens fed diets containing cassava leaf: blood meal mix as replacement for soybean meal. Anim. Prod. Nigeria, 12(1): 212-219.

Otabo FR, Labeyrie V., Duval MF., Mabanza J., Mialoundama. 2016. Diversité variétale de manioc sur la base des nominations vernaculaires des agriculteurs dans 4 bassins de production (Hinda, Loudima, Odziba et Oyo) en République du Congo. J. Appl. Biosci. 104: 9932-9941.

Regnier C. 2011. Valorisation des ressources alimentaires tropicales (feuilles et tubercules) chez le porc. [En ligne]. Université des Antilles et de la Guyane. 349p. Disponible sur: http://ressources.ciheam.org/om/pdf/c37/ 99600024.pdf Page consultée le 9 septembre 2021.

Statview 5.0 2000. SAS Institute, University press of Chicago.

Trompiz J, Gomez A, Rincon H, Ventona M, Bohorquez N, Garcia A. 2007. Effet of rations withcassavaleafflour on productive performance broilers. Revista Científica de la Facultad de Ciencias Veterinarias de la Universidad del Zulia. 17(2) : 143-149. 